\title{
Zero density heavy quark SU(2) gauge theory and the Stefan-Boltzmann limit
}

\author{
Kurt Langfeld*, Norbert Lages, and Hugo Reinhardt \\ Institute for Theoretical Physics, University of Tuebingen, Germany \\ E-mail: kurt.langfeld@uni-tuebingen.de
}

\begin{abstract}
$\mathrm{SU}(2)$ lattice gauge theory is investigated where the traces of the Wilson lines at any lattice point and along each direction is constrained to zero. Hence, each of the lattice configurations possesses a vanishing density of heavy (anti-) quarks. The results are compared with those of pure SU(2) gauge theory which can be interpreted as the grand canonical realization of the heavy quark theory where only the ensemble average of the heavy quark density vanishes.

The static quark anti-quark potential of the constrained theory is obtained from (spatially smeared) Wilson loops at zero temperature. We find that the potential coincides with that of pure $\mathrm{SU}(2)$ gauge theory (without constraints). Hence, the familiar "running" of the lattice spacing with $\beta$ is recovered.
\end{abstract}

XXIIIrd International Symposium on Lattice Field Theory

25-30 July 2005

Trinity College, Dublin, Ireland

${ }^{*}$ Speaker. 


\section{The role of the Polyakov line}

\subsection{Heavy quark physics}

If the quark determinant is calculated for an arbitrary gluon fi eld in the heavy quark limit, the correction to the pure gluonic action density is given by [2]:

$$
S_{\text {heavy }}(x) \propto e^{\frac{\mu-m}{T}} \operatorname{tr} P(x)+e^{\frac{-\mu-m}{T}} \operatorname{tr} P^{\dagger}(x),
$$

where $m$ is the heavy quark mass, $T$ is the temperature, and $\mu$ is the quark chemical potential. $P(x)$ is the Polyakov line, i.e., the product of all time-like links forming a line parallel to Euclidean time.

Taking the derivative of the partition function with respect to the chemical potential provides the expectation value of the baryon density. At vanishing chemical potential, we fi nd

$$
\langle\rho\rangle \propto\langle\mathfrak{I} \operatorname{tr} P(x)\rangle .
$$

In the case of $\mathrm{SU}(2)$, this density vanishes because the trace of any $\mathrm{SU}(2)$ matrix is real. For $S U(N>2),\langle\rho\rangle$ vanishes if the center symmetry is realized: If the center transformation

$$
P(x) \rightarrow P^{\prime}(x)=z P(x), \quad z=\exp \left\{i \frac{2 \pi}{N} m\right\}, \quad-N<m \leq N,
$$

is realized, real part and imaginary parts of $\langle\operatorname{tr} P(x)\rangle$ vanish. While a particular lattice confi guration makes a non-trivial contribution to $\operatorname{tr} P(x)$, the ensemble average causes its expectation value to vanish (see fi gure 2 for an illustration for the case SU(2)). This is no longer the case if $T$ exceeds the critical temperature $T_{c}$ for deconfi nement: In the latter case, the above center symmetry is spontaneously broken. The ground state suggested by the lattice ensembles seems to be populated with heavy quarks of $N$-ality $m_{s}$ (where $m_{s}$ specifi es the realized center sector).

Lattice confi gurations of a standard simulation of pure $S U(N)$ gauge theory with periodic boundary conditions can be interpreted as part of a grandcanonical ensemble where, on average, the density of heavy baryons vanishes. By contrast, if we demand that

$$
\mathfrak{I} \operatorname{tr} P(x)=0
$$

for each confi guration, we sample a canonical ensemble for which the baryon density is identical zero for each ensemble member.

\subsection{Topological sectors of perturbative vacua}

It was pointed out by M. Schaden in [1] that even the Yang-Mills groundstate can fall into topological different classes on a torus. Let us briefly review his arguments.

A perturbative ground state of $\mathrm{SU}(\mathrm{N})$ lattice Yang-Mills theory with Wilson action is given by a link confi guration with

$$
\frac{1}{N} \operatorname{tr} P_{\mu v}(x)=1, \quad \forall x, v>\mu
$$




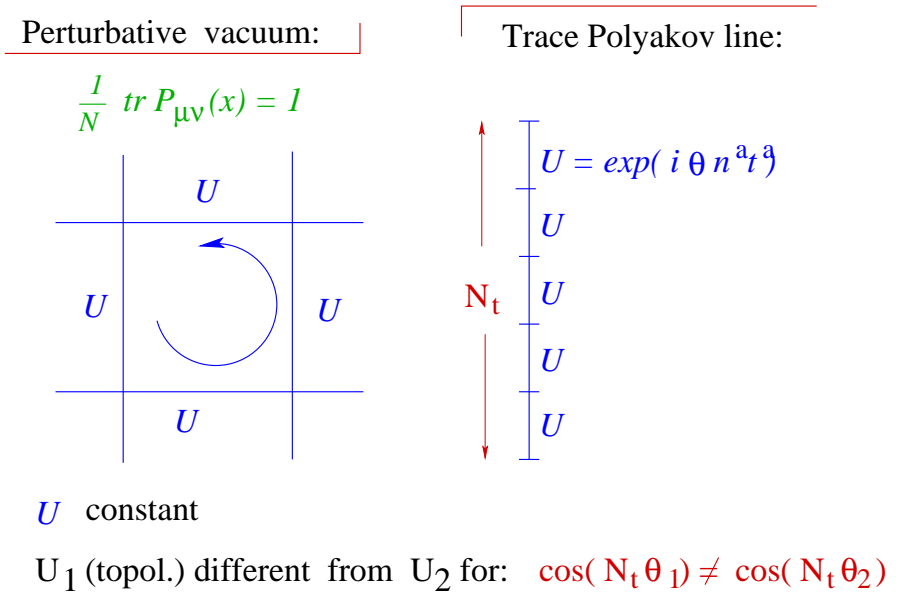

Figure 1: Topological different perturbative sectors on a torus for a SU(2) gauge theory.

where $P_{\mu v}(x)$ is the plaquette built from the links $U_{\mu}(x)$ :

$$
P_{\mu v}(x)=U_{\mu}(x) U_{v}(x+\mu) U^{\dagger} \mu(x+v) U_{v}^{\dagger}(x) .
$$

Obviously, the condition (1.1) is satisfi ed for all constant links

$$
U_{\mu}(x)=U=: \exp \left\{i \theta^{a} t^{a}\right\}, \quad \forall x, \mu
$$

and their gauge equivalents (see fi gure 1). Let us consider the Wilson line $P$ in time direction (Polyakov line) for a lattice with $N_{t}$ links in time direction. Since the trace of the Polyakov line is gauge invariant for a lattice gauge theory with periodic boundary conditions, the perturbative vacua, specifi ed by $U$, can be classifi ed by

$$
\kappa(U)=\frac{1}{N} \operatorname{tr} P=\frac{1}{N} \operatorname{tr} \exp \left\{i N_{t} \theta^{a} t^{a}\right\} .
$$

In particular, two candidates $U_{1}$ and $U_{2}$ are topological inequivalent if $\kappa\left(U_{1}\right) \neq \kappa\left(U_{2}\right)$.

\section{Constrained Yang-Mills theory}

\subsection{Theory}

In the following, we will show results from a simulation of $S U(2)$ and $S U(3)$ lattice gauge theory on a 4-torus using lattice confi gurations with periodic boundary conditions. The lattice confi gurations are subjected to the constraints:

$$
\begin{array}{rlrl}
\operatorname{tr} P(\vec{x}) & =0, & \forall \vec{x} & \text { for } S U(2) \\
\mathfrak{I} \operatorname{tr} P(\vec{x})=0, & \forall \vec{x} & \text { for } S U(3) .
\end{array}
$$

Results will be shown for temperatures below and above the deconfi nement temperature.

The results are interesting for the following reasons: (i) The results will show whether YMtheory with heavy quark fluctuations suppressed (i.e. constrained YM-theory) yields the same results as pure Yang-Mills theory with periodic boundary conditions. Due to spontaneous center 
symmetry breaking at high temperatures, differences are expected at least for the deconfi nement phase. (ii) If constrained and standard YM-theory yield the same physics at least in the confi nement phase, the range of applicability of Schaden's center symmetric perturbation theory [1] might be even extended to $\mathrm{SU}(2)$ gauge theory. (iii) If constrained YM-theory is confi ning, the confi nement mechanism might well be in the reach of a semi-classical treatment of the partition function based upon background fi elds with particular holonomies such as calorons [3, 4].

To be specifi c, the partition function of the simulation is e.g. in the SU(2) case given by

$$
Z=\int \mathscr{D} U \prod_{\vec{x}} \delta(\operatorname{tr} P(\vec{x})) \exp \left\{S_{\mathrm{Wil}}\right\}
$$

One might ask whether the constraints in the $\delta$-functions affect the bulk properties of the lattice ensemble at all: $N_{t}$ link elements contribute to a given Polyakov line. Hence, $3 N_{t}$ real numbers (for SU(2)) must satisfy one constraint. This seems to suggest that the constraint is irrelevant in the infi nite volume limit. Note, however, that $N_{t}-1$ links can be gauged to unity. In addition, the remaining link can be chosen diagonal (up to a gauge transformation). This implies that in lattice gauge theory, there is only one physical degree of freedom contained in the $N_{t}$ links of the Polyakov line. From this arguments, we expect a signifi cant influence from the constraints in (2.3).

\subsection{Numerical setup}

Firstly, a local update algorithm on the basis of M. Creutz heat bath algorithm was implemented. There, a particular link was chosen for the update, and the update of this link was performed according to its probabilistic weight, properly taking into account the constraint.

satisfying changing the trace of the Polyakov line. Seemingly, this overemphasizes the constraints, and one might fear that the algorithm is not ergodic.

For this reason, we secondly constructed an algorithm where all links, "participating" in one particular Polyakov line, are updated simultaneously. We found that both algorithms yield the same results (at least for the observables shown below). Hence, ergodicity is not a problem for the present algorithms.

\section{Numerical results}

In a fi rst step, we have calculated the static quark anti-quark potential at zero temperatures by means of (spatially) smeared Wilson loops. Our fi ndings for the constrained case are summarized in fi gure 2. The striking result is that, despite the constraints, the potential is still linear rising at large distances. In addition, the potential agrees for each $\beta$ separately with the potential from standard simulations. This implies that the scaling, i.e., the dependence of the lattice spacing with $\beta$, is unchanged by the constraints. In addition, the potential was obtained for different orientations of the quark anti-quark pair relative to the cubic lattice ((110), (110), (110) directions). Our result shows that breaking of rotational symmetry by the underlying lattice is small.

Subsequently the question arises whether constrained YM-Mills theory possesses a deconfi nement phase transition at high temperatures despite the constraints which set the Polyakov line 

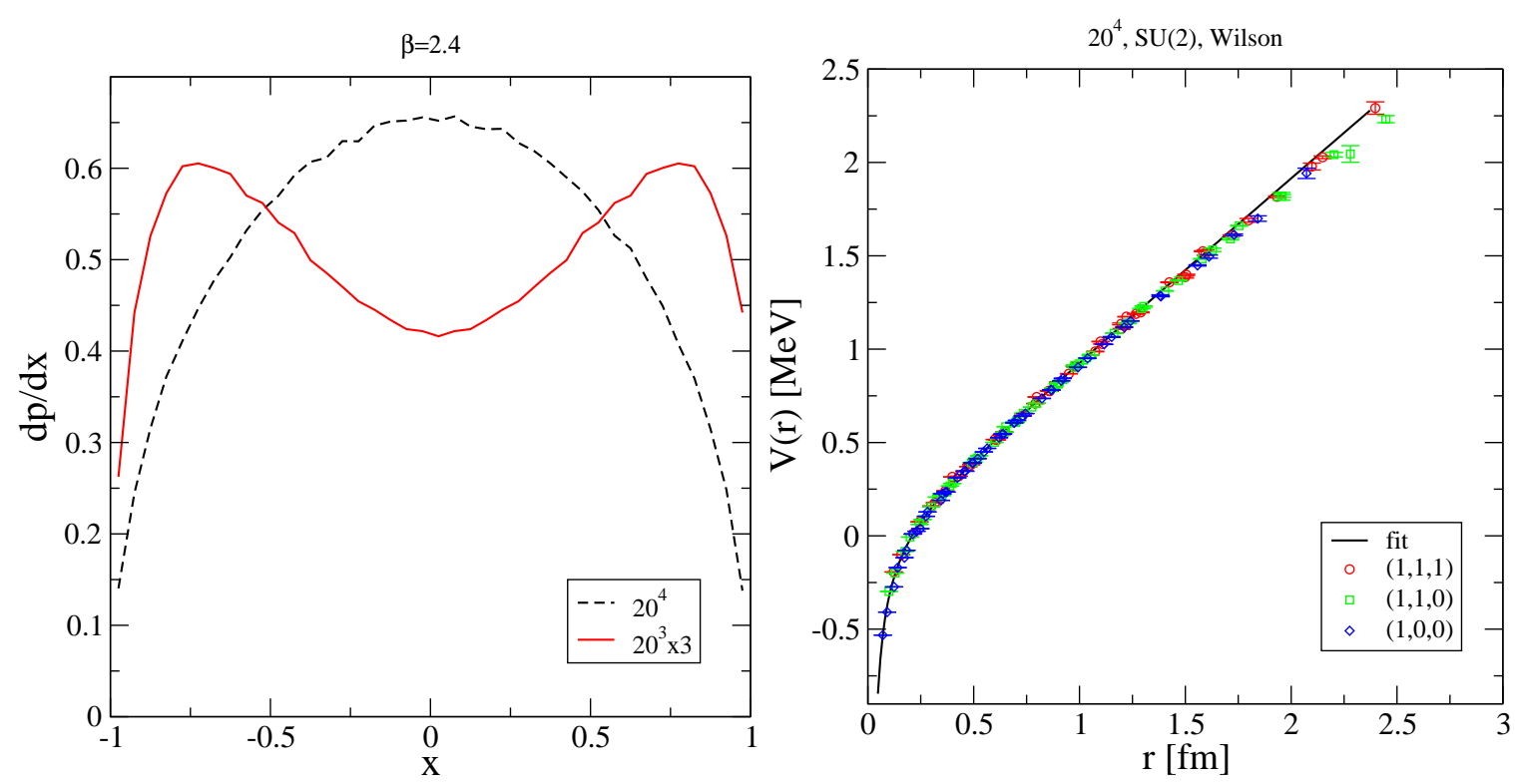

Figure 2: SU(2): Distribution of the Polyakov line expectation values for temperatures below and above the deconfinement one (left panel). Static quark anti-quark potential as function of the quark anti-quark distance for constrained YM-theory at zero temperature (right panel).
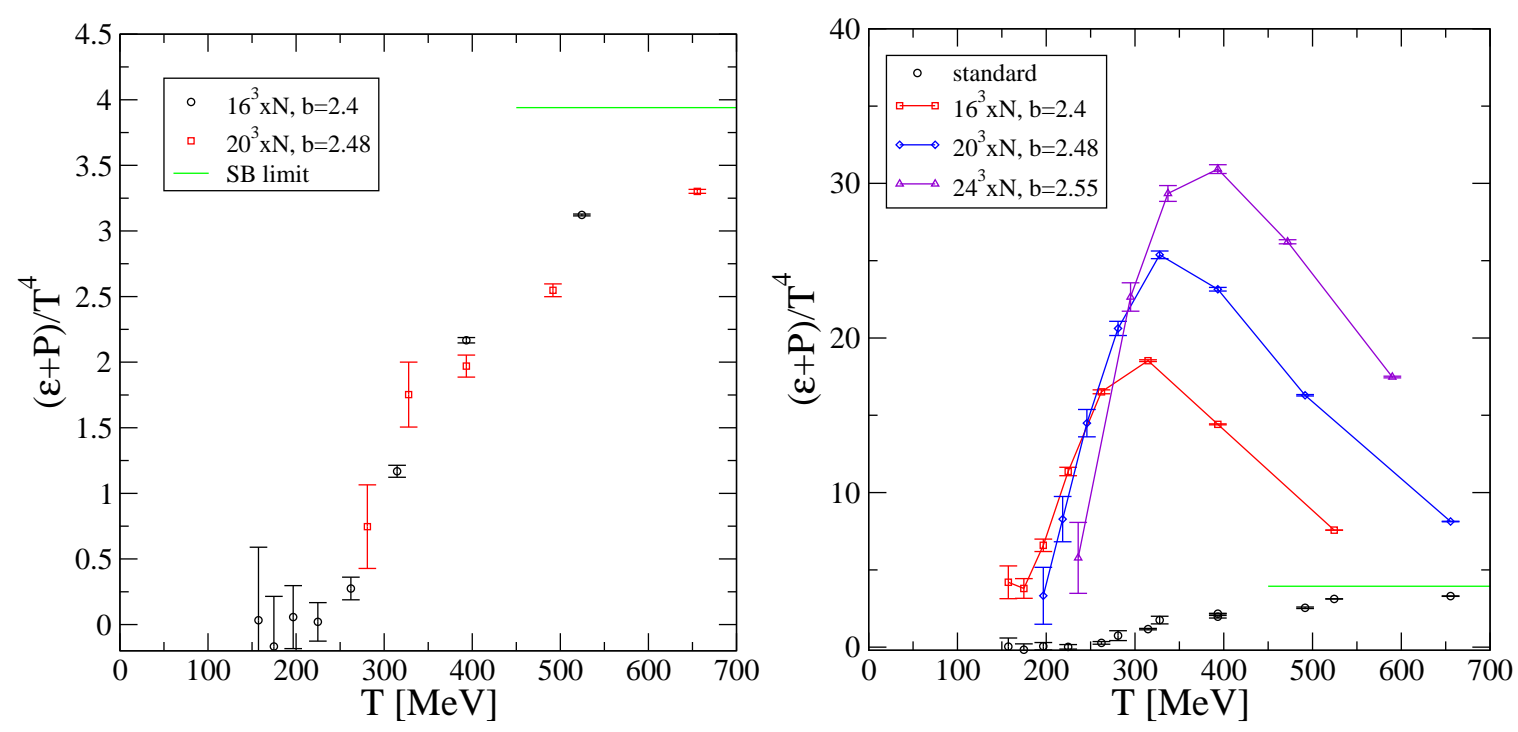

Figure 3: $\mathrm{SU}(2)$ : the sum of energy density $\varepsilon$ and pressure $p$ as function of temperature.

expectation value to zero. In order to answer this question, we have monitored the "black body radiation" of gluons as function of temperature. In particular, we have calculated

$$
\frac{\varepsilon+p}{T^{4}}=f(\beta) N_{t}^{4}\left(P_{s}-P_{t}\right)
$$

where $\varepsilon$ is the energy density and $p$ is the pressure, $P_{s}, P_{t}$ are space-like and time-like plaquettes, respectively, and $f(\beta)$ is a scaling function [5]. Simulations were performed at fi xed $\beta$ while temperature was varied by changing $N_{t}$. Our result for standard SU(2) YM-theory is shown in fi gure 3 

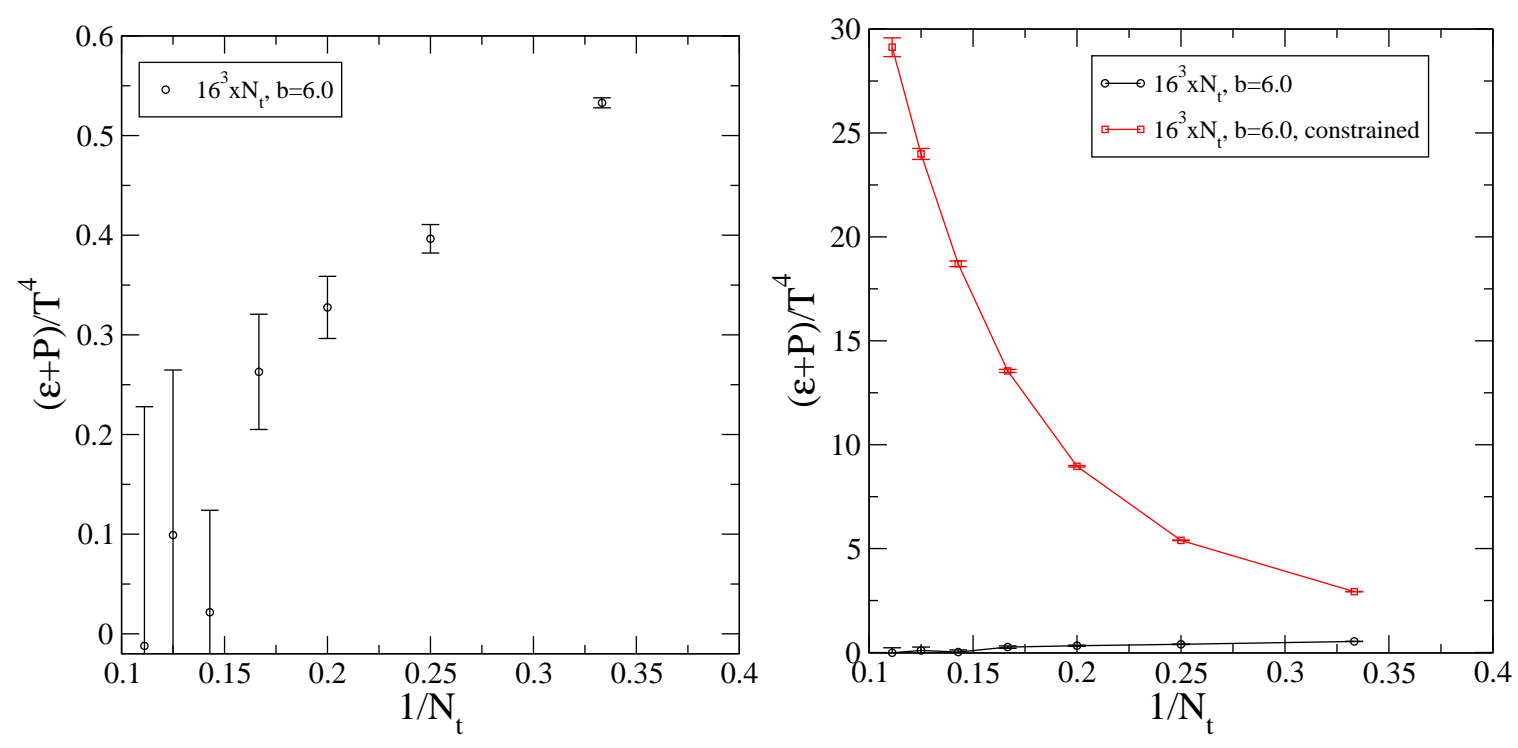

Figure 4: Same as figure 3 for the case of SU(3): standard SU(3) (left), constrained SU(3) (right).

and agrees with the well-known results of the Bielefeld group [5]: The onset of deconfi nement is signaled by a sharp rise of $\varepsilon+p$. Figure 3 also shows our result for constrained YM-Theory. We fi nd also sharp rise of $\varepsilon+p$ at roughly the correct deconfi nement temperature $\mathfrak{T}$. In addition, $\varepsilon+p$ strongly peaks at $T_{c}$ and seem to approach the Stefan Boltzmann limit from above. Unfortunately, we observe a strong cutoff dependence of $\varepsilon+p$ above $T_{c}$, which renders a physical interpretation of the results cumbersome.

Let us fi nally study the case of a SU(3) gauge theory with constraint (2.2). In fi gure 4, the result for $\varepsilon+p$ of standard YM-theory (left panel) is compared with that obtained from simulations of the constrained theory. In the constrained case we fi nd that $\varepsilon+p$ is monotonically decreasing with temperature. No signal of a deconfi nement transition is observed. Moreover, it seems that black body radiation from deconfi ned gluons is present at all temperatures. For an interpretation of these fi ndings, it is important to notice that $\mathrm{SU}(3)$ center symmetry is broken by the constraints. Also center symmetry is merely associated with quark confi nement, there seems also no confi nement of gluons if center symmetry is broken.

Acknowledgment: This work was supported in part by DFG-Re 856/5-1.

\section{References}

[1] M. Schaden, Phys. Rev. D 71, 105012 (2005) [arXiv:hep-th/0410254].

[2] K. Langfeld and G. Shin, Nucl. Phys. B 572, 266 (2000) [arXiv:hep-lat/9907006].

[3] T. C. Kraan and P. van Baal, Phys. Lett. B 435, 389 (1998) [arXiv:hep-th/9806034].

[4] F. Bruckmann, D. Nogradi and P. van Baal, Nucl. Phys. B 698, 233 (2004) [arXiv:hep-th/0404210].

[5] J. Engels, F. Karsch and K. Redlich, Nucl. Phys. B 435, 295 (1995) [arXiv:hep-lat/9408009]. 